\title{
Los Requisitos Funcionales de los Registros Bibliográficos (FRBR), su relación con las RDA y los cambios que se avecinan
}

\author{
The Functional Requirements for Bibliographic Records (FRBR), its \\ Relationship with the GDR and the Changes that lie ahead
}

\author{
Loirette Calvo Sánchez * \\ Escuela de Bibliotecología, Documentación e Información, Universidad Nacional
}

Recibido: 31 de agosto 2016 Aceptado: 14 de noviembre 2016

Corregido: 26 de noviembre Publicado: 05 de mayo 2017

\begin{abstract}
Resumen
El campo de la organización de la información está pasado por una transformación acelerada, debido a la explosión de la información, a la diversidad de soporte y al desarrollo de los entornos web, lo que ha dado lugar a una serie de cambios en el tratamiento de la información. Por consiguiente, esta investigación pretende identificar en qué consiste el modelo de entidad-relación propuesto por la IFLA denominado FRBR (Requisitos Funcionales de los Registros Bibliográficos), cuáles son los principios de catalogación y cuáles son los cambios plasmados en las RDA (Descripción y acceso a los recursos en relación con las RCAA2).
\end{abstract}

Palabras clave: Requisitos funcionales de los registros bibliográficos (FRBR), Descripción y Acceso a Recursos, Declaración de Principios Internacionales de Catalogación, Tratamiento de la Información.

\begin{abstract}
The field of the information organization is going through an accelerated transformation due to the explotion of the information, to the diversity of support and to the development of the web environments, what has given place to a series of change in the treatment of information. Consequently this investigation pretends to identify what constitutes the model of related identity proposed by the IFLA denominated FRBR (Functional Requirements for Bibliographic Records). What are the Principles of Paris, Cataloging International Principles and in what way influences them in the drafting of the RDA and which are the changes reflected in the RDA (Resources Description \& Access) in relation with the AACR2.
\end{abstract}

Keywords: Functional Requirements for Bibliographic Records (FRBR), Resource Description and Acces (RDA), Principles of Paris, Cataloging International Principles. 


\section{Introducción}

El tema de RDA es ampliamente abordado a nivel internacional y nacional por académicos y catalogadores desde que se inició el proceso de su creación. Sin embargo, la intención de esta investigación es hacer una revisión bibliográfica usando la técnica de análisis de contenido que permita identificar algunos de los cambios propuestos en RDA. Además, se busca generar una guía que permita ofrecer una visión general a estudiantes de bibliotecología y a catalogadores sobre los cambios que propone dicho código de catalogación en relación con las Reglas de Catalogación Angloamericanas, 2ª ed. (RCAA2), la incorporación de algunas de las etiquetas del Formato MARC 21 Bibliográfico, los cambios establecidos en la terminología y el modelo de entidad-relación establecido en los Requisitos Funcionales de los Registros Bibliográficos (FRBR).

Para abordar este tema es necesario establecer un referente teórico que permita comprender cuáles son los aspectos que originan el nuevo Código de Catalogación (RDA), su relación con la Declaración de Principios Internacionales de Catalogación y la identificación de los tres grupos de entidades que describe el modelo FRBR.

\section{Las RDA y las RCAA2}

La primera publicación de las Reglas de Catalogación Angloamericanas (RCAA1) para la descripción bibliográfica fue en 1967 y ha tenido diversas actualizaciones a lo largo de la historia hasta llegar a la (RCAA2). Según Escamilla (1978) la segunda edición de este código de catalogación fue pensada para permitir la automatización de los registros catalográficos, favoreciendo la cooperación internacional, con el fin de generar e intercambiar información bibliográfica. No obstante, el avance tecnológico, la diversidad de soportes y las formas de acceso a la información exigieron la actualización de las reglas para satisfacer los requerimientos de la sociedad de la información y dar paso a una nueva normativa internacional denominada por su acrónimo en inglés RDA (Resource and Description Access) y en español Descripción y Acceso a Recursos. 
De acuerdo con Azaña (2011) "en las últimas décadas el universo de la información ha tenido diversos cambios de una manera vertiginosa y constante" (p.29). Tradicionalmente los recursos que almacenaban las bibliotecas estaban en formato impreso y su tratamiento se realizaba usando procesos manuales como la elaboración de fichas catalográficas. Posteriormente, con el uso de la tecnología, esos registros se almacenaron en computadoras que permitieron la automatización de los catálogos disponibles en bibliotecas (OPAC) y el desarrollo del formato de intercambio de información MARC 21 para la transferencia de registros. Actualmente es posible la creación de un nuevo código de catalogación RDA que responde a las necesidades de información. Vargas y Zamorano (2012) señalan que los RDA se basan en los siguientes documentos:

1. La nueva Declaración de Principios Internacionales de Catalogación para su aplicación a los catálogos en línea de las bibliotecas que sustituye y amplía el alcance de los Principios de París.

2. Los Requisitos Funcionales de los Registros Bibliográficos (FRBR) que consiste en un modelo entidad-relación que permite representar conceptos y relaciones e incursionar en una representación dinámica de los actuales OPAC.

3. Los Requisitos Funcionales para Registros de Autoridad (FRAD) que describen las entidades asociadas a un recurso que se conocen como persona, entidad corporativa y familia.

\section{a. Declaración de Principios Internacionales de Catalogación}

Esta declaración es conocida también como Principios de París, fue publicada en 1961 y "...ha servido como base para la normalización internacional en la catalogación y la mayoría de los códigos que se desarrollaron en el mundo" (IFLA 2009 p. 1). Debido a la evolución de las tecnologías de la comunicación y la información, los entornos digitales dinámicos y las nuevas necesidades de información por parte de la comunidad de usuarios, ha sido necesario hacer una revisión de los Principios de París. 
En 2009, la Federación Internacional de Asociaciones de Bibliotecarios e Instituciones (IFLA) presenta la nueva Declaración de Principios Internacionales de Catalogación que comprende "principios generales, entidades, atributos, relaciones, objetivos, funciones del catálogo, descripción bibliográfica, puntos de acceso y fundamentos para las capacidades de búsqueda" (IFLA, 2009 p.1):

- Los principios generales están relacionados con el interés del usuario en los catálogos respecto al uso común del vocabulario, representación, precisión, necesidades, significancia de los datos, economía en las búsquedas, normalización e integración en la descripción de todo tipo de materiales.

- Las entidades, atributos y relaciones se refieren al modelo conceptual FRBR que responde al nuevo código de catalogación RDA.

- Los objetivos y funciones del catálogo es permitir al usuario encontrar, identificar, seleccionar un recurso bibliográfico, adquirir un ejemplar del documento buscado y navegar por un catálogo que permita relacionar las obras, expresiones, manifestaciones, ítem, creadores, conceptos, objetos, acontecimientos y lugares.

- La descripción bibliográfica está basada en estándares internacionales y la elección de puntos de acceso de obras y expresiones.

- Se deben crear puntos de acceso autorizados para la recuperación de datos bibliográficos y de autoridad, con el fin de proporcionar una búsqueda y recuperación fiable de registros bibliográficos y de autoridad. (IFLA, 2009).

\section{b. Requisitos Funcionales para Registros Bibliográficos (FRBR)}

De acuerdo con el grupo de estudio de la IFLA (2004) sobre FRBR declara que este es un modelo conceptual que establece relaciones entre los distintos grupos de entidades y sus conceptos, dando como resultado una nueva forma de descripción bibliográfica. Este modelo entidad-relación del FRBR dividió las entidades en tres grupos para desarrollar el modelo conceptual en el que están basadas las RDA:

$\checkmark$ El primer grupo incluye los productos de creación intelectual o artística que se describen en los registros bibliográficos: obra, expresión, manifestación e ítem. 
$\checkmark$ El segundo grupo comprende aquellas entidades responsables del contenido intelectual o artístico, la producción y difusión física o la custodia de dichos productos: personas, entidades corporativas y familias.

$\checkmark$ El tercer grupo abarca un conjunto adicional de entidades que sirven como sujetos de una producción artística o intelectual: concepto, objeto, acontecimiento y lugar. (IFLA 2004)

Las entidades del primer grupo están representadas por las necesidades del usuario en cuanto a la localización de un recurso o un creador de una obra determinada: obra, expresión, manifestación e ítem. Estas cuatro entidades están interrelacionadas entre sí, la obra está asociada al potencial intelectual del autor, seguido por la expresión que se refiere a la realización intelectual de esa obra que se representa a través de una traducción o película de una obra, etc. La manifestación se identifica como el soporte en el cual se almacena esa expresión y por último un ítem de la manifestación ubicado en cualquier unidad de información (Figura 1).

Figura 1

Representación de la entidad del grupo 1

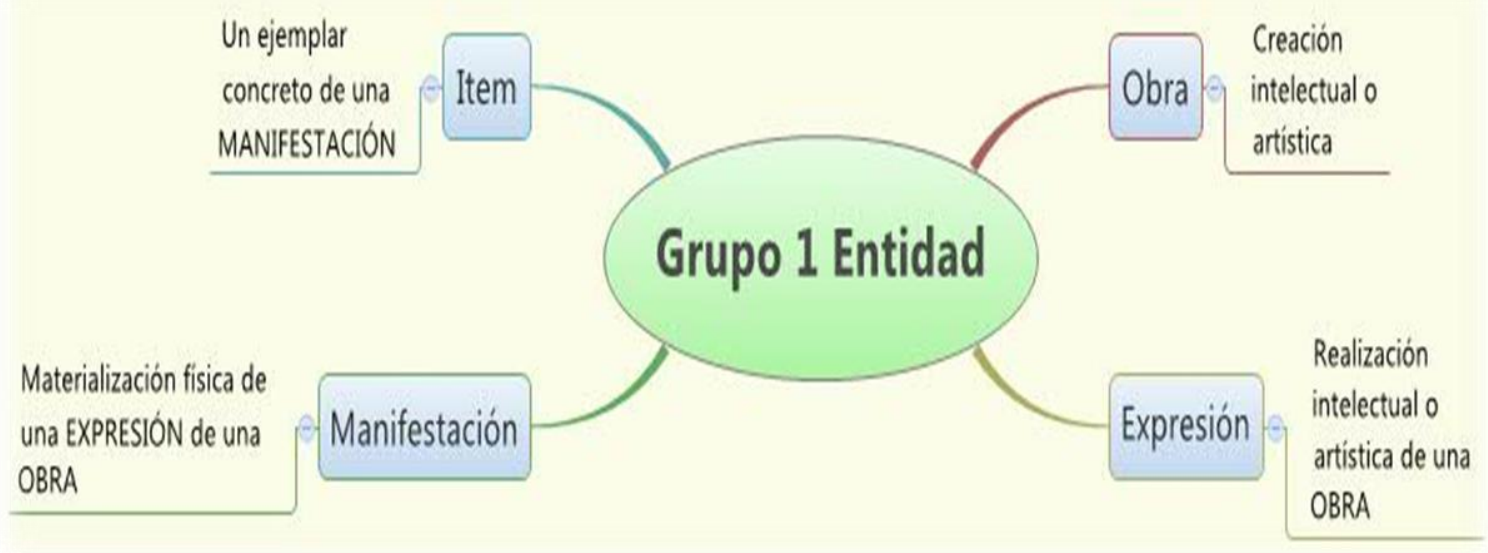

Fuente: Calvo, L. (2015). 
Las entidades del segundo grupo incluyen autores o grupos de personas o entidades corporativas, responsables de una obra. "Las entidades del segundo grupo incluyen persona (un individuo) o entidad (una organización o grupo de individuos y/o organizaciones) y familias". (IFLA, 2004, p.53) Este grupo no representa elementos nuevos de la tradición catalográfica que se han trabajado a lo largo de la historia, como autores personales, corporativos denominados en las RDA como creadores de las obras (Figura 2).

\section{Figura 2}

\section{Representación de las entidades del grupo 2}

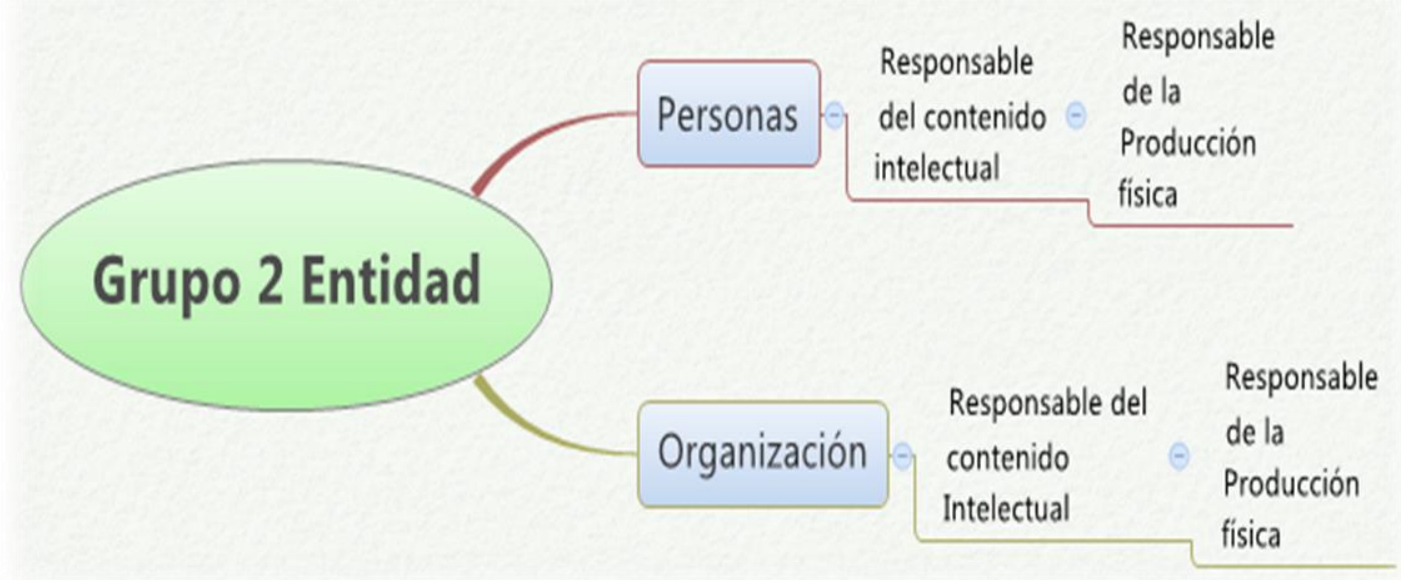

Fuente: Calvo, L. (2015).

En el tercer grupo de entidades (conceptos, objetos, acontecimientos y lugares), IFLA (2004) "incluye concepto (una idea o noción abstracta), objeto (una cosa material), acontecimiento (una acción o suceso) y lugar (una localización)" (p. 54). El concepto se refiere a la materia, campos del conocimiento o disciplina de una obra, por ejemplo filosofía, derechos humanos o bibliotecología.

Para la entidad definida como objeto se podría citar el Teatro Nacional de Costa Rica. La entidad definida como acontecimiento incluye acciones, sucesos acontecimientos históricos o épocas, por ejemplo, Siglo XX o Batalla de Santa Rosa. La entidad definida como lugar abarca localizaciones o representaciones geográficas tales como Costa Rica, Guatemala, 
Plaza de San Pedro en Roma. Cada uno de estos elementos se considera entidades solo si constituyen la materia de una obra. La Figura 3 representa las cuatro entidades del grupo tres que se usan para representar las materias de una obra.

Figura 3

\section{Representación de las entidades del grupo 3}

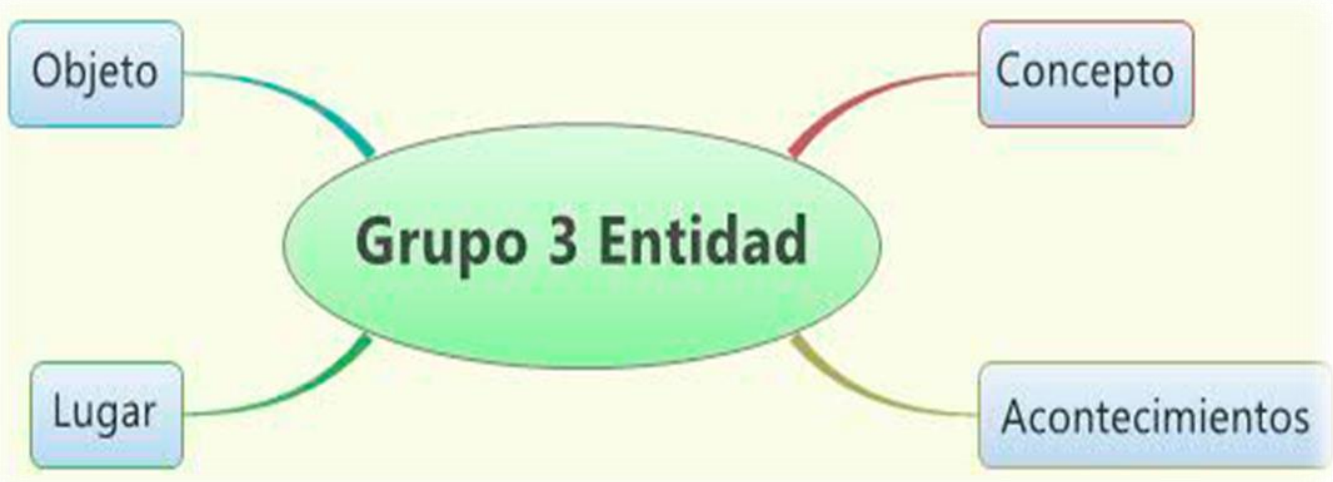

Fuente: Calvo, L. (2015).

El modelo conceptual entidad-relación del FRBR es la base de RDA para explicar el universo bibliográfico. Según González (2011) "en términos simples, se puede decir que es un modelo que identifica cosas y cómo estas cosas se relacionan entre si" (p.2). Las RDA, junto con el modelo FRBR y el FRAD, instauran un nuevo paradigma en el tratamiento de la información que se adaptada a la creación y al uso de los catálogos en línea, recursos digitales y la visualización por medio de la web semántica.

\section{Resultados encontrados en la revisión bibliografía}

\section{a. Relación entre RCAA2 y RDA}

Las RCAA2 se diseñaron para la descripción bibliográfica y actualmente no responden adecuadamente a las necesidades de descripción y recuperación de la información de una diversidad de soportes. Según lo explica Vargas y Zamorano (2012) "Las RCAA2 han sido 
revisadas y actualizadas para adaptarse a los nuevos formatos, pero en esencia fueron creadas para los catálogos de fichas" (p.13).

Es por ello que surgen las RDA con la finalidad de proporcionar una herramienta digital e impresa que se adapte a las nuevas formas de acceder a la información, a diversidad de formatos, a las nuevas tecnologías y a un entorno de trabajo basado en la web que desarrolla actualmente las unidades de información, para satisfacer las necesidad de los usuarios.

Martínez (2009) señala que "el nuevo código de catalogación, RDA, no es algo totalmente nuevo puesto que éste ha sido desarrollado teniendo en cuenta la experiencia de las RCAA2" (p. 4). Por consiguiente, hay cambios significativos en las RDA en cuanto a su organización y distribución, la cual se establece por secciones, según los tres grupos de entidades presentados en el modelo FRBR y no por capítulos y tipos de materiales como se presenta en las RCAA2. Es aquí donde radica el principal cambio en la organización de esta normativa internacional.

\section{b. Diferencias entre la estructura de las RCAA2 con las RDA}

El capítulo 1 de RCAA2 incluye reglas generarles para la descripción bibliografía. A partir del capítulo 2 están orientadas a los diversos tipos de materiales (libros, material cartográfico, manuscritos, música, grabaciones sonoras, películas y videograbaciones, material gráfico, recursos electrónicos, artefactos tridimensionales, realia, microformas, recursos continuos) y por último el capítulo 13 está asignado a las analíticas. Cada capítulo dicta las reglas para la descripción de los diversos tipos de materiales con sus respectivas fuentes prescritas de información.

A partir del capítulo 21 se pueden encontrar las reglas para la elección de los puntos de acceso como asientos principales y secundarios. El capítulo 22 para los encabezamientos de personas, el 23 para nombres geográficos, el 24 para encabezamientos de entidades 
corporativas, el 25 para la creación de títulos uniformes y por último el 26 para la creación de referencias. Por consiguiente, la primera parte está relacionada con la descriptiva, basada en la norma de Descripción Bibliográfica Internacional Normalizada (ISBD), y la parte dos se concentra en la elección y forma del asiento.

Las RDA se organizan en secciones y no sigue la estructura de las RCAA2 que divide la descripción por tipo de materiales y la elección de puntos de acceso, identificado la puntuación prescrita por áreas. Las secciones de las RDA están estructuradas siguiendo el modelo conceptual que se establecen entre los tres grupos de entidades que constituyen el FRBR.

A continuación se intentará hacer una comparación básica entre los capítulos de RCAA2 y las secciones de las RDA sin ánimo de hacer una amplia explicación de cada una de las secciones, pero sí que permita a los estudiantes y catalogadores construir una relación lógica entre ambas:

a. La sección uno (Registro de atributos manifestación e ítem) de las RDA se relaciona con la descripción de materiales del capítulo 2 al 12 de la RCAA2.

b. La sección dos (Registro de atributos de obras y expresiones) se puede comparar con el capítulo 25 de títulos uniformes que se usa para agrupar varias expresiones de una obra en una colección determinada en una unidad de información. En las RDA se llama título preferido.

c. La sección tres (Personas, familias y entidades corporativas) se puede homologar con el capítulo 22 de encabezamientos de personas y el capítulo 25 de encabezamientos de entidades corporativas.

d. La sección cuatro (Registro de atributos de conceptos, objetos, eventos y lugares y el registro de relaciones). No está representada en ninguno de los capítulos de las RCAA2. Esta se desarrollará junto con la sección diez de relaciones después de la primera publicación de las RDA. 
e. Las secciones cinco, seis, siete, ocho y nueve indican las pautas para establecer los registros de relaciones entre obras, expresiones, manifestaciones, ítems, personas, familias y entidades corporativas y es acá donde se refleja el cambio más significativo entre las RDA y las RCAA2.

Las secciones que contienen las RDA incluyen una serie de atributos que deben ser tomados en consideración para encontrar, identificar, seleccionar y obtener un recurso y las entidades asociadas al mismo.

\section{c. Atributos del grupo de entidades uno y dos}

En el Cuadro 1 y 2 se pueden identifican los atributos básicos que están presentes en los dos grupos de entidades desarrollados hasta este momento con las pautas establecidas en las RDA y las etiquetas Marc.

\section{Cuadro 1}

\section{Atributos de la Entidad del grupo uno}

\section{OBRA}

1. Título preferido de la obra (véase RDA 6.2.2) $=240 \$ a$

2. Forma de la obra (véase RDA 6.3) $=380 \$ a$

3. Fecha de la obra (la fecha más antigua asociada con la obra) (véase RDA 6.4)= $388 \$ a$

4. Lugar de origen de la obra (véase 6.5)=

5. Otra característica distintiva de la obra (véase RDA 6.6)

6. Historia de la obra $=$

\section{EXPRESIÓN}

1. Término que indica el tipo de contenido (véase RDA 6.9) 336\$a

2. Fecha de la expresión (La fecha de la expresión es la fecha más antigua asociada con una expresión). (véase RDA 6.10) no MARC

3. Lengua de la expresión (véase RDA 6.11) 377\$a

4. Otra característica distintiva de la expresión (véase RDA 6.12)

\section{ÍTEM}

1. Identificador del ítem (Número de adquisición) =

2. Fecha de adquisición =

3. Método de adquisición (compra, canje, donación) =

4. Custodia del ítem (Biblioteca que tiene el ítem) = 


\section{MANIFESTACIÓN}

2. Título propiamente dicho (véase RDA 2.3.2) $245 \$ a$

3. Información complementaria del título (véase RDA 2.3.4) 245\$b

4. Título paralelo propiamente dicho (véase RDA 2.3.3) $245 \$ b$

5. Mención de responsabilidad (si hay más de una, solo se requiere la primera registrada) (véase RDA 2.4) 245\$c

6. Mención de edición (véase RDA 2.5) $250 \$ a$

7. Lugar de publicación (si hay más de uno, solo se requiere el primero registrado) (véase RDA 2.8.2.2) $264 \$ a$

8. Nombre del editor (si hay más de uno, solo se requiere el primero registrado) (véase RDA 2.8.4.2) $264 \$ b$

9. Fecha de publicación (véase RDA 2.8.6.2) 264 \$c

10. Fecha del copyright (véase RDA: 2.11)

11. Título propiamente dicho de la serie (véase RDA 2.12.2.2) $490 \$ a$
1. Mención de responsabilidad relacionada con la serie, (véase RDA 2.12.6.2) \$a

2. Numeración dentro de la serie (véase RDA 2.12.9.2) \$v

3. ISSN de la serie, (véase RDA 2.12.8.2) \$x

4. Título propiamente dicho de la subserie (véase RDA 2.12.10.2) \$a

5. Numeración dentro de la subserie (véase RDA 2.12.17.2) \$v

6. Identificador de la manifestación (si hay más uno, elija un identificador reconocido internacionalmente si fuera aplicable ejemplo ISBN) $020 \$ a$

7. Tipo de soporte (véase RDA 3.3) $338 \$ a$

8. Tipo de medio (véase RDA 3.2) $337 \$ a$

9. Extensión (véase RDA 3.4) $300 \$ a$

10. Ilustraciones (véase RDA 7.15 y 7.17 .2 a color) $300 \$ a$

11. Modo de edición (véase RDA 2.13 unidad individual, monografía en varias partes, publicación seriada, recurso integrable) campo fijo 008 posición 07

Fuente: elaboración propia a partir de RDA: Recursos, Descripción y Acceso, (2015)

En este cuadro se ejemplifica los atributos que se deben considerar en cada una de las entidades del grupo uno del RFBR. La obra y la expresión toma los atributos que están presentes en la sección dos, la manifestación y el ítem de la sección uno de las RDA.

En el Cuadro 2 se aprecia la inclusión de una serie de atributos que permiten construir una diferencia entre los diversos creadores de un recurso, elementos que no estaban presentes en las RCAA2, pero pueden ser consultados en la sección tres de RDA: registro de atributos de persona, familia y entidad corporativa. El cambio en la estructura de la RDA por secciones también está acompañado por una serie de cambios en la terminología y en la 
descripción que resultan importantes identificar para poder comprender la conceptualización que está presente en esta normativa.

\section{Cuadro 2}

\section{Atributos de la entidad del grupo 2 Creadores}

\begin{tabular}{|c|c|}
\hline 046 Fechas especiales codificadas & 371 dirección (RDA 9.10, 9.11) \\
(RDA 9.3.2 y 9.3.3) & 372 Campo de Actividad (R) (RDA \\
100 Autor Personal (RDA 9.2.2 y 19.2) & 9.15 ) \\
368: Otros atributos de personas o & 373 Afiliación - Grupo Asociado (R) \\
entidades corporativas (R) & (RDA 9.13) \\
370: Lugar asociado & 374 Ocupación (R) (RDA 9.17) \\
\$a Lugar de nacimiento (NR) (RDA & 375 Género (R) (RDA 9.7) \\
9.8) & 377 Lengua asociada (R) (RDA 9.14) \\
\$b Lugar de fallecimiento (NR) (RDA & 378 Forma más completa del \\
9.9) & Nombre Personal (NR) \\
& 400 -Referencia de envió "véase"- \\
& nombre variante de la persona \\
& (RDA 9.2.3.10) \\
\hline
\end{tabular}

Fuente: elaboración propia a partir de RDA: Recursos, Descripción y Acceso, (2015)

\section{Cambio en la terminología y la descripción entre RCAA2 y RDA}

Es oportuno identificar una serie de cambios en la terminología que contemplan las RDA en relación con las RCAA2. En el Cuadro 3 se muestra una comparación de los términos.

Algunos de los cambios en la terminología reflejan el alejamiento del entorno de catálogos de fichas, a un entorno digital. El término encabezamiento y asiento principal tenía sentido cuando se elaboraban fichas bibliográficas para los catálogos manuales, en los cuales las RCAA2 proporcionan un conjunto de normas o lineamientos que se aplican a la descripción bibliográfica y que proveen la forma que deben adoptar los encabezamientos. 


\section{Cuadro 3}

Cambio en la Terminología entre RCA2 y RDA

\begin{tabular}{|c|c|}
\hline AACR2 & RDA \\
\hline Encabezamiento & Punto de acceso autorizado \\
\hline Autor, compositor, artista, etc. & Creador \\
\hline Entrada principal & $\begin{array}{l}\text { Título preferido y, cuando sea apropiado, punto de } \\
\text { acceso autorizado para el creador }\end{array}$ \\
\hline Título uniforme & $\begin{array}{l}\text { Título preferido y cualquier información diferente; } \\
\text { un título colectivo convencional como "Obras" }\end{array}$ \\
\hline Referencia véase & Diferentes puntos de acceso \\
\hline Referencia véase además & Punto de acceso autorizado para entidad relacionada \\
\hline Descripción física & Descripción soportes \\
\hline $\begin{array}{l}\text { Designación General del } \\
\text { Material DGM }\end{array}$ & $\begin{array}{l}\text { Tres elementos nuevos a considerar } \\
\text { 1. tipo de contenido } \\
\text { 2. tipo de medio } \\
\text { 3. tipo de soporte }\end{array}$ \\
\hline Modo de edición & $\begin{array}{l}\text { Unidad individual } \\
\text { Monografía en varias partes } \\
\text { Publicación seriada } \\
\text { Recurso integrable }\end{array}$ \\
\hline Fuente principal & Fuentes preferidas \\
\hline
\end{tabular}

Fuente: Biblioteca del Congreso (2013).

Hay otros cambios que se establecen en RDA que resulta adecuado destacar, entre ellos se pueden señalar:

$\checkmark$ Principio de representación que significa tome lo que ve. Esto permite que se describan los atributos de una manifestación tal como se presenta en el recurso. Para facilitar la captura de datos y que los usuarios puedan encontrar, identificar, seleccionar y obtener un recurso determinado.

$\checkmark$ No se usa más [sic] ni [i.e] en los errores detectados en las monografías (Cuadro 4). 


\section{Cuadro 4}

Corrección de errores uso del [sic] ni [i.e]

\begin{tabular}{|c|c|}
\hline RCAA2 & RDA \\
\hline $\begin{array}{c}24504 \text { \$a Los derechos de las } \\
\text { mujres [i.e. mujeres] }\end{array}$ & $\begin{array}{c}24504 \text { \$a Los derechos de las } \\
\text { mujeres }\end{array}$ \\
& $24600 \$$ i Título Corregido \$a \\
derechos de las mujeres
\end{tabular}

Fuente: Elaboración propia de la autora.

Se elimina la regla de tres que consistía en registrar como máximo tres autores en un registro, lo cual tenía sentido en la confesión de fichas bibliográficas. En RDA se pueden registrar los autores que el centro catalográfico considere necesario o agregar entre paréntesis el resto de autores.

$\checkmark$ En la mención de responsabilidad se elimina la regla de tres y, por consiguiente, la abreviatura [et al.] que significa y otros más, como se muestra en el Cuadro 5.

\section{Cuadro 5}

Comparación Eliminación de la Regla de tres

\begin{tabular}{|c|c|}
\hline RCA2 & RDA \\
\hline \multirow{8}{*}{$\begin{array}{l}24500 \quad \$ a \text { Introducción a la } \\
\text { investigación de mercados } / \$ c \\
\text { María Jesús Merino Sanz... [et } \\
\text { al.]. }\end{array}$} & $245 \quad 10 \quad$ Introducción a la \\
\hline & investigación de mercados $/ \$ c$ \\
\hline & María Jesús Merino Sanz, Teresa \\
\hline & Pintado Blanco, Joaquín Sánchez \\
\hline & $\begin{array}{lll}\text { Herrera y Ildefonso Grande } \\
\text { Fsteban }\end{array}$ \\
\hline & Omisión opcional: \\
\hline & $245 \quad 10 \$$ \$a Introducción a la \\
\hline & $\begin{array}{l}\text { María Jesús Merino Sanz [y otros } \\
\text { tres]. }\end{array}$ \\
\hline
\end{tabular}

Fuente: Elaboración propia de la autora. 
En RCAA2 para registrar los datos de publicación se usan abreviaturas para indicar que el recurso no registra editorial y lugar de publicación. En RDA se eliminan abreviaturas y se sustituyen por palabras completas. La misma situación ocurre con la descripción física como se muestra en el Cuadro 6.

\section{Cuadro 6}

\section{Datos de publicación y descripción física}

\begin{tabular}{|c|l|}
\hline RCA2 & \multicolumn{1}{c|}{ RDA } \\
\hline 260\#\# \$a[S.I. :\$bs.n.],\$c2012. & $\begin{array}{l}264 \# 1 \$ a[\text { Lugar de publicación no } \\
\text { identificado] :\$b[Editor no identificado],\$c2012 }\end{array}$ \\
\hline 300\#\# \$a200 p :\$bil (algunas col.) & $\begin{array}{l}300 \# \$ a 200 \text { páginas :\$bilustraciones } \\
\text { (algunas a color) }\end{array}$ \\
\hline
\end{tabular}

Fuente: Elaboración propia de la autora.

La Designación General del Material (DGM) que se registraba en el campo 245 subcampo (\$h) donde se indicaba el tipo de material, se elimina en RDA de la descripción y se incorporan los siguientes campos:

- Tipo de contenido de la obra que se está describiendo.

- Tipo de medio que se requiere para tener acceso al recurso.

- Tipo de soporte donde se almacena el recurso.

La implementación de las RDA también implica cambios para el formato Marc 21 Bibliográfico, como se puede observar en los ejemplos del Cuadro 7.

\section{Cuadro 7}

\section{Designación General del Material (DGM)}

\begin{tabular}{|l|l|}
\hline \multicolumn{1}{|c|}{ RCA2 } & \multicolumn{1}{c|}{ RDA } \\
\hline Designación General & - Tipo de Contenido [336]: sonido, texto, etc. \\
del Material (DGM) & - Tipo de Medio [337]: audio, video, sin mediación etc. \\
Ej.: [videograbación], & - Tipo de Soporte [338]: volumen, casete de audio, \\
[diapositiva], etc. & diapositiva, videodisco, etc. \\
\hline
\end{tabular}




\begin{tabular}{|l|l|}
\hline Ejemplo: & Ejemplo: \\
10010 \$a Gutiérrez & 10010 \$a Gutiérrez Mangel, Joaquín, \$d 1918-2000\$e \\
Mangel, Joaquín, \$d & autor \\
$1918-2000$ & $24510 \$ a$ Cocorí / \$c Joaquín Gutiérrez \\
$24510 \$ a$ Cocorí \$h & $336 \# \$$ atexto\$btxt\$2rdacontent \\
[texto] / \$c Joaquín & (Tipo de contenido) \\
Gutiérrez & $337 \# \#$ \$a sin mediación \$bn\$2 rdamedia \\
& (Tipo de medio) \\
& $338 \# \#$ \#a volumen $\$ b n c \$ 2$ rdacarrier \\
& (Tipo de soporte sin mediación) \\
\hline
\end{tabular}

Fuente: Elaboración propia de la autora.

Como se ha mencionado a lo largo del documento, las RCAA2 se diseñaron para la descripción bibliográfica y la organización de los datos bibliográficos en ocho áreas, tal como lo establece la norma ISBD, caracterizada por su puntuación y la aplicación de abreviaturas en la elaboración de sus registros.

Con la aplicación de las RDA se podrían señalar algunos cambios en la construcción de los registros en los OPAC:

1. Se construyen los registros centrados en el usuario.

2. Se eliminan abreviaturas que únicamente eran comprendidas por los bibliotecólogos y se descarta la reglas de tres que tenía sentido en los catálogos manuales.

3. Se usa formatos de intercambio de información que mejor se adapten a cada comunidad de usuarios ( ${ }^{1}$ ISBD, ${ }^{2}$ Dublin Core, ${ }^{3}$ Marc y ${ }^{4}$ RDF).

4. Se establece una estructura por sección según los tres grupos de entidades.

5. Se aplica el modelo conceptual en la construcción de relaciones entre los grupos de entidades, lo cual representa uno de los cambios más significativos en RDA y permite cumplir con las tareas del usuario de encontrar, identificar, seleccionar y obtener un recurso por medio de un catálogo más dinámico e interactivo, basado en entornos web. 
Bibliotecas. Vol 35, № 1, enero-junio, 2017, pp.1-23. EISSN: 1659-3286

URL: http://www.revistas.una.ac.cr/index.php/bibliotecas/index

\section{Relaciones entre los grupos de entidades}

En la Figura 4 se ejemplifican las relaciones que existen en el primer grupo de entidades: Obra, Expresión, Manifestación e Ítem identificados en las RDA.

\section{Figura 4}

\section{Ejemplos de las entidades del grupo 1}

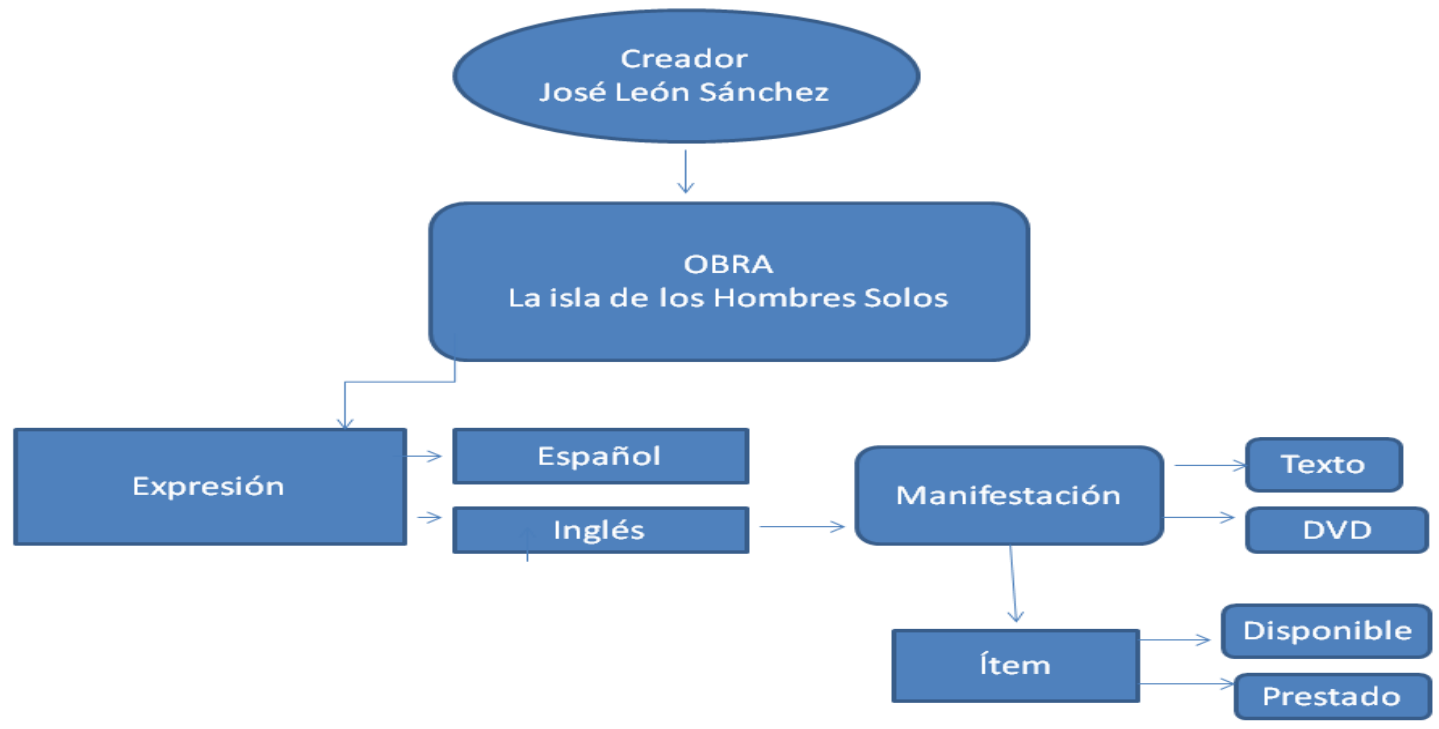

Fuente: Elaboración propia de la autora.

Una obra es una creación intelectual o artística que se genera a partir de la imaginación e intelecto del creador. La expresión es la realización de una obra en forma de texto, música, coreografía, sonido, imagen, etc. Se podría indicar que cualquier cambio en el contenido intelectual o artístico constituye un cambio de expresión. Si se revisa o modifica un texto, por ejemplo una traducción de una lengua a otra, la expresión resultante se considera una nueva expresión, independientemente de que se trate de una modificación menor". (IFLA, 2004 p. 61), por ejemplo:

a. La obra La Isla de los hombres solos de José León Sánchez.

b. La Expresión es el texto en inglés o la película La Isla de los hombres solos. 
La manifestación es la realización física de la expresión de una obra. Las manifestaciones se encuentran en una biblioteca en diversos soportes físicos o digitales que oscilan desde un manuscrito hasta una publicación digital. Para retomar el ejemplo anterior, las manifestaciones de esta obra se materializarían en un (texto impreso o en línea del libro La isla de los hombres solos o la película del director René Cardona, soportadas en un CD o DVD). Por tanto, la manifestación se refiere al medio por el que se ha comunicado el contenido de una obra.

Por su parte, el ítem es definido por IFLA (2004) "como entidad que permite identificar independientemente ejemplares individuales de una manifestación" (p. 68). Un ítem es un ejemplar determinado de una manifestación que puede tener diferentes estados en una biblioteca, disponible, prestado o perdido. Además, deben relacionar las entidades del grupo 1 con las del grupo 2 tal como se representan en el Cuadro 8.

\section{Cuadro 8}

\section{Relaciones entre la Entidad 1 y 2}

\begin{tabular}{|ccccc|}
\hline $\begin{array}{c}\text { Entidad } \\
\text { del Grupo }\end{array}$ & Obras & Expresión & Manifestación & \\
1 & & & & \\
Entidad & Creadores & Colaboradores & Distribuidor de la & Propietario o \\
del Grupo & Ejemplo el & de la Obra & manifestación & Custodio del \\
2 & Autor de & Ejemplo el & ejemplo una & ítem Ejemplo \\
& la obra & Traductor de la & editorial & una biblioteca \\
& & obra expresada. & & \\
\end{tabular}

Fuente: elaboración propia a partir de RDA: Recursos, Descripción y Acceso, (2015)

La sustitución de las RDA por RCAA2 no es un simple cambio en la descripción. Implica más elementos como incorporar las nuevas etiquetas de la familia Marc 21 bibliográfico y de autoridad, y comprender cómo se aplica el modelo conceptual entidad - relación. Para hacer un ejercicio visual sobre la aplicación de las relaciones entre los grupos de entidades se 
Bibliotecas. Vol 35, № 1, enero-junio, 2017, pp.1-23. EISSN: 1659-3286

URL: http://www.revistas.una.ac.cr/index.php/bibliotecas/index

sugiere visitar la página http://www.marcofquality.com/wiki/rimmf3/doku.php?id=download y descargue de forma gratuita el Rimmf3.exe.

\section{Imagen 1}

\section{Visualización de Rimmf3}

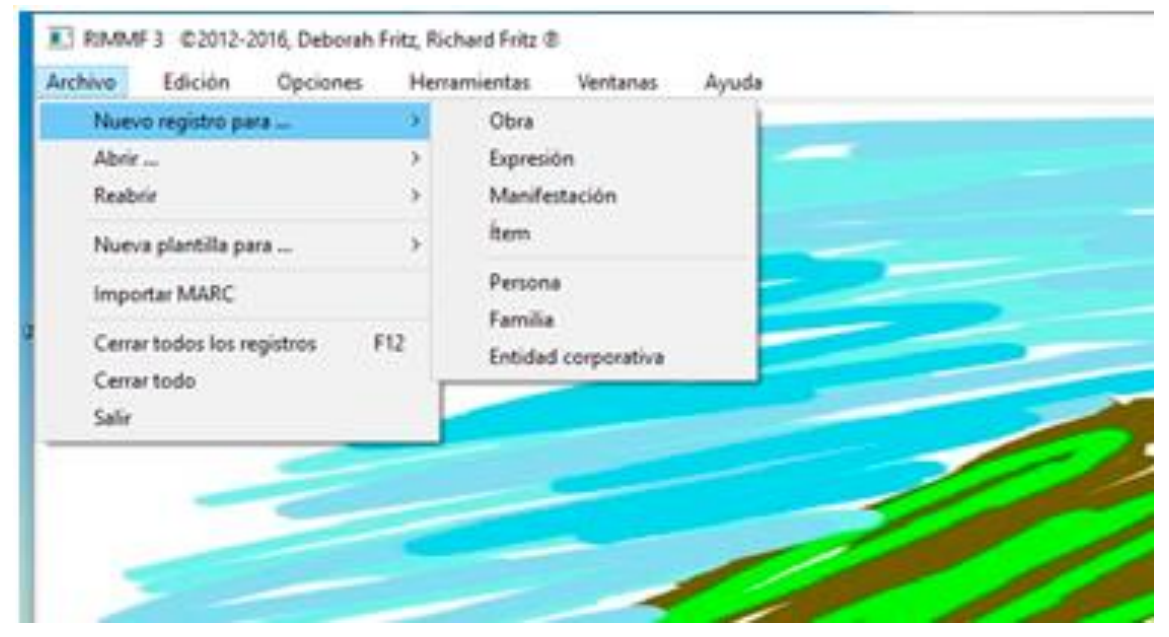

Fuente: Deborah F. y Richard F. (2016).

Esta es una herramienta de visualización que puede ayudar a entender los atributos de las RDA y las relaciones que se gestan entre las entidades del grupo uno y dos del FRBR.

Se sugiere que se inicie el uso de esta aplicación en el siguiente orden:

- Identifique los atributos de la manifestación, obras, expresiones e ítems. No olvide que debe construir las relaciones con el grupo dos (persona, familia y entidad corporativa) del modelo entidad-relación.

\section{Conclusiones}

Como lo han expuesto algunos autores las RDA tienen sus orígenes en la aplicación de las RCAA2 y en la experiencia de los grupos que han participado en la redacción de este nuevo código de catalogación. Si se analiza con detenimiento los cambios establecidos en relación con el uso de la nueva terminología y la eliminación de las abreviaturas, tiene sentido en el 
momento en el que inicia la automatización de los registros, donde el término encabezamiento pierde su sentido de aplicación. El uso de las abreviaturas y la regla de tres tenían una justificación lógica cuando se realizaban fichas bibliográficas para ficheros manuales, donde el espacio era reducido y la duplicación de trabajo era interminable.

El cambio de las RCAA2 a las RDA es necesario y la comunidad bibliotecológica debe iniciar por los elementos más sencillos: eliminar las abreviaturas, la regla de tres, e incorporar las nuevas etiquetas que sustituyen la designación general de material (DGM) y la construcción de los catálogos de autoridad de autor. Se debe iniciar negociaciones con los proveedores de sistemas de información para incorporar las nuevas etiquetas del formato MARC 21 Bibliográfico y de autoridad que permita realizar las relaciones entre los tres grupos de entidades.

Sin lugar a duda, la aplicación del modelo entidad-relación de FRBR para el establecimiento de relaciones es el cambio más significativo en RDA. El elemento más complejo de comprender es cómo puede aplicarse en los sistemas de información para poder establecer las relaciones entre las entidades para que la comunidad de usuarios pueda encontrar, identificar, seleccionar y obtener una obra, expresión, manifestación e ítem determinado.

La aplicación real de este modelo está en proceso y pasarán algunos años para que el desarrollo del formato Biframe en RDF esté listo y se puedan ver los resultados en los sistemas de información existentes en el mercado y al alcance de la comunidad latinoamericana.

Por el momento se podría usar el Rimmf3.exe para comprender mejor el modelo entidadrelación y realizar prácticas que permitan a los catalogadores y estudiantes una mejor visualización de los atributos que están presentes en los dos grupos de entidades y como estos se relacionan entre sí. 


\section{Referencias}

Azaña, S.C. (2011). RDA: ¿Dar una nueva norma o una nueva concepción de catalogación? $\checkmark$ encuentro de catalogadores. México: UNAM.

Calvo, L. (2015). Utilización de herramientas colaborativas para el diseño de una guía didáctica digital para los cursos de procesos técnicos de la Escuela de Bibliotecología y Ciencias de la Información de la Universidad de Costa Rica. Tesis de maestría en Bibliotecología. Universidad de Costa Rica, San José: Costa Rica.

González, E. (2011). RDA: un acercamiento a las nuevas normas de catalogación. Revista Chilena de Bibliotecología (2) 2-14. Chile.

Deborah F. y Richard F. (2016). Rimmf user guide. Recuperado de http://www.marcofquality.com/wiki/rimmf3/doku.php?id=download\&nocache

IFLA (2009). Declaración de principios internacionales de catalogación. Recuperado de http://www.ifla.org/files/assets/cataloguing/icp/icp 2009-es.pdf

IFLA (2004). Requisitos Funcionales de los Registros Bibliográficos Informe final. Recuperado de http://www.ifla.org/files/assets/cataloguing/frbr/frbr-es.pdf

Library of Congres (2013). Capacitación LC sobre RDA Recursos: Descripción y Acceso.

Recuperado de http://www.loc.gov/catdir/cpso/RDA/RDAcapacitacionLC.html

Library of Congres (2011). Bibliographic Framework Initiative. Recuperado de http://www.loc.gov/bibframe/

Martínez, F. (2009). Las RDA (Resource Description and Access) y su impacto en la catalogación y los catálogos: necesidades de investigación. Recuperado de http://132.248.242.3/ publica/archivos/libros/iv encuentro catalogacion.pdf

Piñuela, J. (2002). Epistemología, metodología y técnicas de análisis de contenido. Estudio de sociolingüística, 3 (1) 1-42. Madrid: Universidad Complutense de Madrid.

Reglas de catalogación angloamericanas: Antecedentes y resumen (1978). Trad. G. Escamilla González. México: Conacyt

RDA: Recursos, Descripción y Acceso, (2015). American Library Association. Colombia. Rojas Eberhard Editores. 
Vargas, C. y Zamorano, A. (2012). La RDA ¿Mito o realidad?: Panorámica de la catalogación en Chile. Serie Bibliotecología y Gestión de Información, 72. Santiago, Chile: Universidad Tecnológica Metropolitana.

\section{Notas de la autora}

* Loirette Calvo Sánchez: académica de la Escuela de Bibliotecología, Documentación e Información, Universidad Nacional, Costa Rica. Correo electrónico loirethc@gmail.com

${ }^{1}$ ISBD es la norma que determina los elementos y el orden específico de los datos que se deben registrar en el recurso que se está catalogando, siguiendo una puntuación prescrita.

${ }^{2}$ Dublin Core está compuesto por un conjunto de elementos repetibles usados para describir un amplio abanico de recursos en red.

${ }^{3}$ El formato Marc es una serie de campos, subcampos, indicadores que son usados para registrar los atributos de los recursos y tiene como objetivo el intercambio de los registros.

${ }^{4}$ El modelo de datos RDF (Resource Description Framewor) usa un enfoque de modelado conceptual como entidad-relación y puede ser usado para describir las relaciones entre las entidades. 